Macedonian Pharmaceutical Bulletin, 66 (Suppl 1) 175 - 176 (2020)

Online ISSN $1857-8969$

UDC: $616.311 .2-003.2-085.281$

DOI: 10.33320/maced.pharm.bull.2020.66.03.087

Short communication

\title{
Collection, sample preparation and analytical methods for determination of therapeutic levels of drugs in gingival crevicular fluid - a review
}

\author{
Liljana Anastasova $^{1} *$, Mirjana Popovska ${ }^{2}$, Rumenka Petkovska $^{1}$ \\ ${ }^{1}$ Institute of Applied Chemistry and Pharmaceutical Analysis, Faculty of Pharmacy, \\ Ss. Cyril and Methodius University, 1000 Skopje, Republic of North Macedonia \\ ${ }^{2}$ Department of Oral Pathology and Periodontology, Faculty of Dentistry, Ss. Cyril and Methodius University, \\ 1000 Skopje, Republic of North Macedonia
}

\section{Introduction}

Gingival crevicular fluid (GCF) is an inflammatory exudate containing substances from the host (enzymes, cytokines, interleukins or tissue breakdown products), as well as from supra- and subgingival located bactreria (Wassall \& Preshaw, 2016). It plays a significant role in the oral defense mechanism and facilitates transport of antibacterial substances into the periodontal pocket. Literature findings imply that certain drugs used in the treatment of periodontal disease can accumulate in gingival fibroblasts and serve as reservoirs which gradually release the substance in GCF. Therefore, GCF concentrations of certain drugs can be used as an indicator of treatment response (Dincel et al., 2005; Lai et al., 2011). This review will provide an overview of the methods for GCF collection, sample preparation, commonly used analytical methods for quantitative determination of drug concentration in GCF as well as future developments in this area.

Collection methods and sample preparation techniques for GCF samples

The collection of GCF samples is regarded as noninvasive and relatively simple procedure without having to take a sample from gingival tissue.
However, GCF volumes are typically low (microliter quantities) and generally increase with increasing inflammation in gingival and periodontal tissues. Therefore, the quantity and quality of GCF samples can be highly affected by the method of collection and analysis. The GCF sampling procedure has been performed using a variety of methods, including gingival washing techniques, capillary tubes or micropipettes as well absorbent filter paper strips. The most frequently used technique is the filter paper method (Wassall \& Preshaw, 2016). In the last decade, researchers have favored the filter paper strips due to easy insertion into a gingival crevice up to $1 \mathrm{~mm}$ depth without bleeding from periodontal pockets (Khurshid et al., 2017)

GCF is a complex matrix especially in inflamed sites where the protein composition resembles the serum (Papagerakis et al., 2019). In order to minimize the interferences that could arise in the determination of the drug concentration in GCF, prior analysis liquid-liquid extraction usually followed by vortexing or centrifugation is performed. In this manner, the drug of interest is eluted in the supernatant and ready for subsequent analysis (Dincel et al., 2005; Sagan et al., 2005).

\footnotetext{
*lbogdanovska@ff.ukim.edu.mk
} 


\section{Methods for quantitative determination of drugs in GCF samples}

For the quantitative determination of drug concentrations in GCF several analytical methods have been employed: gas chromatography (GC), high-performance liquid chromatography (HPLC) as well as high-performance liquid chromatography coupled with mass detection (HPLC-MS).

GC analysis has been applied for determination of nifedipine which is sequestrated into GCF causing drug-induced gingival overgrowth. The main disadvantage of this "scenario" is the derivatization step in the sample preparation procedure in order to make it suitable for GC analysis (Guncu et al., 2007). HPLC is a powerful tool in the analysis of drugs in different biological matrices. It has the ability to separate and identify compounds that can be dissolved in suitable liquid and detect trace concentrations as low as parts per trillion. Also, the wide pallet of detectors available for the HPLC enables determination of different molecules (Dong, 2013). HPLC with UV detection has been used in the analysis of drugs such as metronidazole, tinidazole, phenytoin, spyramycin and other commonly used antibiotics especially in the treatment of aggressive forms of periodontal disease. Another group of commonly applied drugs in periodontal treatment, both locally and orally, are fluoroquinolones and tetracyclines. They are accumulated by fibroblasts in gingival connective tissue which potentially enhances their antimicrobial effectiveness in two ways: by increasing their local concentrations in gingiva and sustaining them for a longer duration, which is a factor that must be considered in order to optimize periodontal antimicrobial chemotherapy (Lavda et al., 2004). Due to the specific chemical structure of these molecules HPLC with fluorescent detection has been used as the method of choice in their analysis. In this manner greater sensitivity is achieved (Dincel et al., 2005; Lavda et al., 2004).

Another recently applied method for quantitative determination of drugs in GCF is LC-MS/MS. Compared to HPLC-UV it requires smaller sample volume, it has better specificity and a higher speed of analysis (Sagan et al., 2005). However, due to the complex equipment and the high cost of analysis, it has not been routinely used for determination of drug concentration in GCF samples.

\section{Conclusion}

The collection and quantitative determination of drugs in GCF will continue to play a significant role for evaluation of treatment response. The developments of modern instruments will facilitate the entire process of GCF analysis at the same time leading to facilitated analysis of novel delivery systems intended for treatment of periodontal disease and related conditions.

\section{References}

Dincel, A., Yildrim, A., Caglayan, F., Bozkurt, A., 2005. Determination of ciprofloxacin in human gingival crevicular fluid by high-performance liquid chromatography. Acta Chrom. 15, 308-314.

Dong, M., 2013. The essence of modern HPLC: Advantages, limitations, fundamentals and opportunities. LCGC North Am. 31, 472-479.

Guncu, G.N., Caglayan, F., Dincel, A., Bozkurt, A., Ozmen, F., Karabulut, E., 2007. Clinical and pharmacological variables as risk factors for nifedipine-induced gingival overgrowth. Aust. Dent. J. 52, 295-299.

Khurshid, Z., Mali, M., Naseem, M., Nejeeb, S., Zafar, M.D., 2017. Human gingival crevicular fluid (GCF) proteomics: an overview. Dent. J. 5, 3-8.

Lai, P., Weiting, H., Jain, N., Walters, J., 2011. Azithromycin Concentrations in Blood and Gingival Crevicular Fluid after Systemic Administration. J. Periodontol. 82, 1582-1586.

Lavda, M., Ester Clausnitzer, E., Walters, J., 2004. Distribution of systemic ciprofloxacin and doxycycline to gingiva and gingival crevicular fluid. J. Periodontol. 75, 1663-1667.

Papagerakis, P., Zheng, L., Kim, D., Said, R., Ehlert, A., Chung, K., Papagerakis, S., 2019. Saliva and gingival crevicular fluid (GCF) collection for biomarker screening, in: Papagerakis, P. (Ed.), Odontogenesis: Methods and protocols, methods in molecular biology. Springer Nature, pp.549-562.

Sagan, C., Salvador, A., Dubreuil, D., Poulet, P., Duffaut, D., Brumpt, I., 2005. Simultaneous determination of metronidazole and spiramycin I in human plasma, saliva and gingival crevicular fluid by LC-MS/MS. J. Pharm. Biomed. Anal. 38, 298-306.

Wassall, R., Preshaw, P., 2016. Clinical and technical considerations in the analysis of gingival crevicular fluid. Periodontol. 2000 70, 65-79. 